\title{
Soft Sensing Based on Probabilistic Neural Network
}

\author{
Qiang Wang ${ }^{1, a}$ \\ 1Dongying Vocationnl College,Dongying,Shandong 257091,China \\ awq213117@163.com
}

Keywords: wavelet packet; probabilistic neural network; soft sensing

Abstract. Aimed at the characteristic of nonlinear and non-stationary of pressure drop, in this article a flow regime identification soft sensing method using wavelet-packet combined with probabilistic neural network is put forward. PNN is used as classier due to its good generalization ability and fast learning capability, case of on-line updating, and sound statistical foundation in Bayesian estimation theory. The features are extracted from the differential pressure fluctuation signals of the air-water two-phase flow in the horizontal pipe and the wavelet packet energy features of various flow regimes are obtained. Then combining the energy features with probabilistic neural network, a new way to identify flow regime by soft sensing is proposed.

\section{Introduction}

Today Gas-liquid two-phase flow widely exists in modern industry production. The two-phase flow and heat transfer character are extremely influence by the flow regimes. The flow regime identification is a hot question of international multi-phase flow field today [1]. So the accurate identification of flow regimes is important for the operation and design of interrelated instruments.

In recent years the technology of neural network and nonlinear signal analysis theory and so on have been used in every field [2]. All these give us a new method for two-phase flow identification. It is well known that a PNN is used as classier due to its good generalization ability and fast learning capability, case of on-line updating, and sound statistical foundation in Bayesian estimation theory. PNN has becomes an effective tool for solving many difficult classification problems in practice [3-4].

A novel method of flow regime identification based on wavelet packet decomposition and wavelet packet decomposition is proposed in this paper. Above all, the collected pressure-difference fluctuation signals are decomposed by the four-layer wavelet packet, and the decomposed signals in various frequency bands are obtained within the frequency domain. Then the wavelet packet energy eigenvectors of flow regimes are established. At last the wavelet packet energy eigenvectors are input into probabilistic neural network and flow regime intelligent identification can be performed. This study provided a new way to identify flow regime by soft sensing.

\section{Probabilistic Neural Network}

PNN is a kind of supervised neural network that is widely used in the area of pattern recognition, nonlinear mapping, and estimation of probability of class membership and likelihood ratios. The original PNN structure by Specht[5] is a network formulation of probability density estimation. PNN has proven to be more time efficient than conventional back-propagation based networks and has been recognized as an alternative in real-time classification problems.

The theory of PNN is simple explained based on two kind patterns. The test sample is $X^{T}=\left[X_{1}, \mathrm{~L}, X_{j}, \mathrm{~L}, X_{p}\right]$ vector whose dimension is $p$. Where the sample either belong to $\theta_{A}$ or 
belong to $\theta_{B}$, that is to say $d(X)=\theta_{A}$ or $d(X)=\theta_{B}$. According to Bayesian least risk rule, the decide-making rule is

$$
\begin{gathered}
d(X)=\theta_{A} \text { if } h_{A} l_{A} f_{A}(X)>h_{B} l_{B} f_{B}(X) \\
d(X)=\theta_{B} \text { if } h_{A} l_{A} f_{A}(X)<h_{B} l_{B} f_{B}(X)
\end{gathered}
$$

In which condition function is

$$
f_{A}(X)=\frac{1}{(2 \pi)^{p / 2} \sigma^{p}} \frac{1}{N_{A}} \times \sum_{i=1}^{N_{A}} \exp \left[-\frac{\left(X-X_{A i}\right)^{T}\left(X-X_{A i}\right)}{2 \sigma^{2}}\right]
$$

The sample can be normalized by next formula

$$
\left(X-X_{A i}\right)^{T}\left(X-X_{A i}\right)=-2\left(X^{T} X_{A i}-1\right)
$$

where

$$
h_{A}=\frac{N_{\mathrm{A}}}{N_{\text {total }}}
$$

And $N_{\text {total }}$ is the total count of training samples. Moreover we may suppose $l_{A}=l_{B}$. If we enter formula ( 2 ) (3)and ( 4 ) to formula ( 1 ) then we can obtain

where

$$
\begin{aligned}
& d(X)=\theta_{A} \text { if } P_{A}(X)>P_{B}(X) \\
& d(X)=\theta_{B} \text { if } P_{A}(X)<P_{B}(X)
\end{aligned}
$$

$$
P_{k}(X)=\sum_{i=1}^{N_{k}} \exp \left(\frac{X^{T} X_{k i}-1}{\sigma^{2}}\right), k=\mathrm{A}, \mathrm{B}
$$

The above is the decide-making rule of two kind cases. For more kind cases, we may select the corresponding identified result of maximum $P_{k}(X)$. When the sample is $m$ kind, the decide-making rule is

$$
d(X)=\theta_{k} \text { if } P_{k}=\max \left\{P_{i}\right\}, i=1,2, \mathrm{~L}, m
$$

Where $k \in N$ and $1 \leq k \leq m$.

In this paper, the identify mode belong to four kind pattern. The architecture of the PNN is shown in Fig.1. The PNN has four layers feed forward network including input layer, pattern layer , summation layer and output layer.The respond function of pattern is

$$
Y_{k j}(X)=\exp \left(\frac{X^{T} X_{k j}-1}{\sigma^{2}}\right)
$$

\section{Wavelet-packet energy}

The wavelet packet method is a more subtle orthogonal decomposition method based on multi-resolution ratio. It can select the frequency band self-adaptively according to the characteristics of the analysed signals and ascertain the resolution ratio of the signals in different frequency bands[6]. The square of the signal's 2 norm is equal to the energy of 


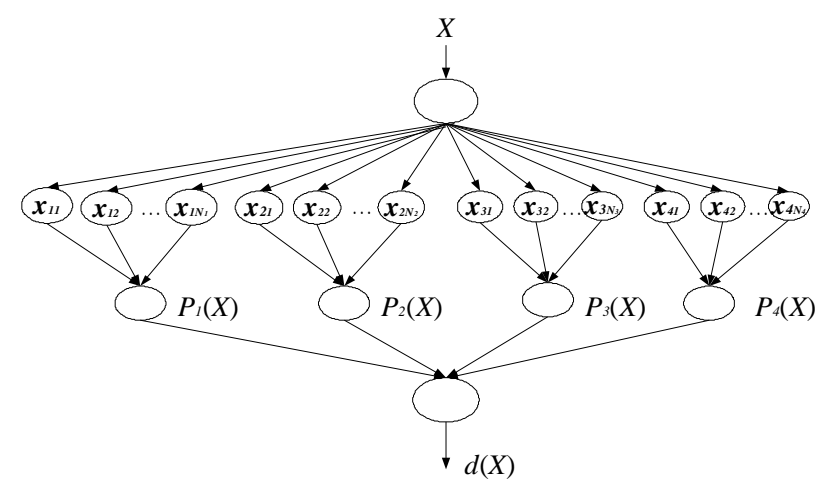

Fig.1 Architecture of the probabilistic neural network

original signal at the time region. So it is reliable that we use wavelet packet energy spectrum to denote the energy of the original signal. The signal of energy may select the sum of the square of various frequency bands. Supposing the corresponding energy of someone frequency bands is $E_{j}$ then we get:

$$
E_{j}=\int\left|S_{j}(t)\right|^{2} d t=\sum_{k=1}^{N}\left|x_{j k}\right|^{2}
$$

Where, $x_{j k}(j=0,1, \mathrm{~L}, 15), k=(0,1, \mathrm{~L}, n)$ represent the discrete points' amplitude of reconstruction signal $S_{j}$ and $\mathrm{N}$ is the sample length of the $\mathrm{j}$ subspace.

The test system introduction and the collection method of the data can be found in literature [7]. In this paper wavelet packet energy is selected as extract feature. Above all, the collected pressure-difference fluctuation signals are decomposed by the four-layer wavelet packet, and the decomposed signals in various frequency bands are obtained within the frequency domain. Then restructure the coefficient of the wavelet packet decomposition, and extract the signals of various frequency bands range(Fig.2 depicted the typical reconstructed signals of various band).Then the wavelet packet energy eigenvectors of flow regimes are established. At last the wavelet packet energy can be normalized by the maximal energy various frequency bands range. Tab.1 represents the test sample data and classification result of four flow-regimes.

\section{Conclusions}

There are identified results of PNN which are parts of the total in table I. From the table we can see that the right identified rate is high with PNN, where 151 samples can be right identified of 160 samples. The whole identification ratio reaches $94.4 \%$. This may also prove that this method is reliable. Moreover comparing with BP neural network PNN is not only high identified velocity but also is high identified rate.

\section{Acknowledgements}

The author are grateful to the support of Project of Shandong Province ( 2015zcj046、J13LD60、 2014kjhm0206) and Project of Teaching Reform. 

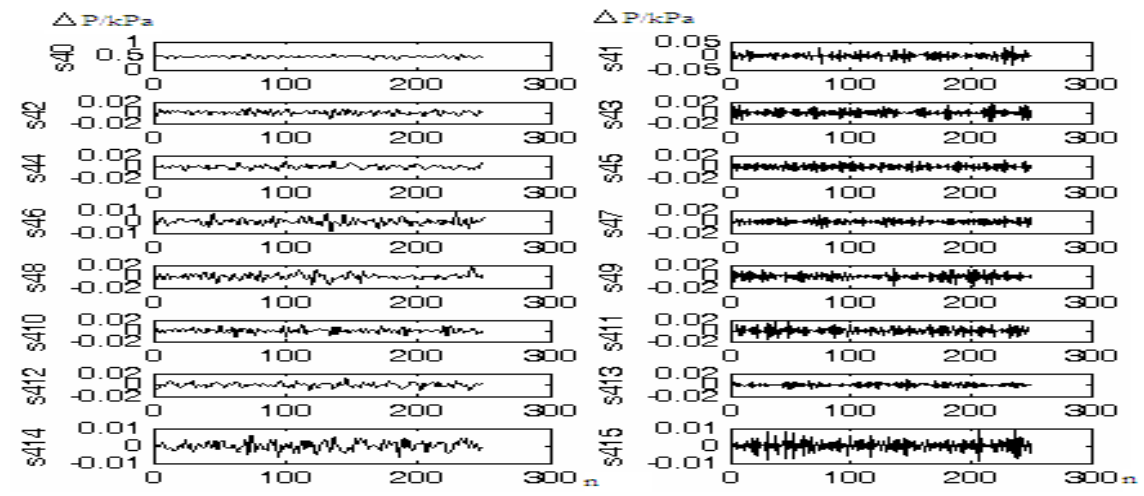

Fig.2 Reconstructed signals of various band

Tab. 1 Test sample data and classification result of four flow regimes

\begin{tabular}{|c|c|c|c|c|c|c|c|c|c|c|c|c|c|c|c|c|}
\hline \multicolumn{16}{|c|}{ Wavelet packet energy feature } & \multirow{2}{*}{$\begin{array}{l}\text { Observing } \\
\text { /PNN } \\
\text { /BP }\end{array}$} \\
\hline $1 \sim 16$ & $16 \sim 32$ & $32 \sim 48$ & $48 \sim 64$ & $64 \sim 80$ & $80 \sim 96$ & $96 \sim 112$ & $112 \sim 128$ & $128 \sim 144$ & $144 \sim 160$ & $160 \sim 176$ & $176 \sim 192$ & 192 208 & $208 \sim 224$ & $224 \sim 240$ & $240 \sim 256$ & \\
\hline 0.76712 & 0.74112 & 0.5697 & 0.51961 & 0.49753 & 0.72123 & 0.60051 & 0.60238 & 0.86314 & 0.55129 & 0.68723 & 0.90609 & 0.63464 & 0.52039 & 0.96732 & 0.51278 & $\mathrm{~B} / \mathrm{B} / \mathrm{B}$ \\
\hline 0.73768 & 0.60865 & 0.41298 & 0.42754 & 0.54483 & 0.67617 & 0.51673 & 0.48892 & 0.88206 & 0.61274 & 0.67696 & 0.82919 & 0.59279 & 0.44725 & 0.83855 & 0.41687 & $\mathrm{~B} / \mathrm{B} / A$ \\
\hline 0.26037 & 0.25264 & 0.41944 & 0.35087 & 0.48717 & 0.90412 & 0.51444 & 0.60065 & 0.83663 & 0.70761 & 0.39705 & 0.81148 & 0.60791 & 0.97097 & 0.60286 & 0.85922 & $\mathrm{I} / \mathrm{I} / A$ \\
\hline 0.27131 & 0.34748 & 0.45773 & 0.47075 & 0.56829 & 0.87707 & 0.53698 & 0.69385 & 0.73919 & 0.68837 & 0.41214 & 0.73596 & 0.59717 & 0.95521 & 0.66413 & 0.79555 & $\mathrm{I} / \mathrm{I} / \mathrm{I}$ \\
\hline 0.11399 & 0.21577 & 0.25513 & 0.20972 & 0.43544 & 0.64623 & 0.29125 & 0.45784 & 0.50311 & 0.38827 & 0.92579 & 0.87427 & 0.61928 & 0.44731 & 0.92484 & 0.39359 & $\mathrm{~S} / \mathrm{S} / \mathrm{S}$ \\
\hline 0.10501 & 0.091 & 0.17651 & 0.08809 & 0.94709 & 0.65053 & 0.42043 & 0.41539 & 0.41803 & 0.92955 & 0.35951 & 0.74261 & 0.97979 & 0.36729 & 0.60233 & 0.38783 & $\mathrm{~A} / \mathrm{A} / \mathrm{A}$ \\
\hline
\end{tabular}

Annotation:S-stratified flow;I-intermittent flow;B-bubbly flow;A-annular flow; Observing- Observing flow patterns

\section{References}

[1] S. Z. Rouhani . "Two-phase flow patters : a review of research results . Nucl . Energy", vol. 11, no.3, pp.219-259.April, 1983.

[2] X. Li, Z.-Q. Chen, Z.-Z. Yuan, et al. "Generating chaos by an Elman network", IEEE Transactions on Circuits and Systems-I: Fundamental Theory and Applications, vol.48, no.9, pp.1126-1131, March,2001.

[3] Y.-D. Gao, Z.-C. Zhang . "Diagnosis of damping faults in helicopter rotor hub based on fuselage vibrations". Transactions of Nanjing University of Aeronautics \&Astronautics. Vol.23, no.2, pp. 102-107, June 2006.

[4] S. Ramakrishnan, S. Selvan "Multiwavelets domain singular value features for image texture classification", Journal of Zhejiang University SCIENCE A, vol.8,no.4, July,2007.

[5] D.-F. Specht. "Probabilistic neural networks", Neural Networks, vol.3, no.1, pp.109-118, July, 1990.

[6] M. Cotronei, L.-B. Montefusco, L. Puccio. "Multiwavelets

analysis and signal processing" IEEE Trans. Circuits Syst. II, vol.45, no.8, pp.970-987.March, 1998.

[7] Q. Wang, Y.-L. Zhou, Y.-F. Cui, et al. "Applied study of EMD and neural networks on flow regime identification for gas-liquid two-phase flow", Journal of Engineering Thermophysics, vol.28, no.3, pp.442-444, June,2007. 